\title{
Antiretroviral drug resistance and HIV-1 subtypes among treatment-naive prisoners in Kelantan, Malaysia
}

\author{
Tengku Ahmad Akram Tengku Mohd Ariffin ${ }^{1}$, Suharni Mohamad ${ }^{2}$, Wan Nazirah Wan Yusuf ${ }^{3}$, Rafidah \\ Hanim Shueb ${ }^{1}$ \\ ${ }^{1}$ Department of Medical Microbiology and Parasitology, Universiti Sains Malaysia, Kubang Kerian, Kelantan, \\ Malaysia \\ ${ }^{2}$ School of Dental Sciences, Universiti Sains Malaysia, Kubang Kerian, Kelantan, Malaysia \\ ${ }^{3}$ Department of Pharmacology, Universiti Sains Malaysia, Kubang Kerian, Kelantan, Malaysia
}

\begin{abstract}
Introduction: The widespread use of highly active antiretroviral therapy (HAART) and continuous reports of HIV-1 strains developing resistance to these drugs is rather alarming, as transmission of resistant viruses to newly infected persons is possible. This study aimed to determine HIV-1 subtypes and the prevalence of primary mutations associated with antiretroviral (ARV) resistance among treatment-naive prisoners on the east coast of Malaysia.

Methodology: Viral RNA was extracted from plasma samples of 21 treatment-naive prisoners. Protease (PR) and reverse transcriptase (RT) regions were amplified and sequenced. Stanford HIV database algorithms were used for interpretation of resistance, and phylogenetic analysis was performed for subtype assignment.

Results: In the PR gene, no antiviral resistance-associated mutation was detected. For RT-associated mutations, K103N was the most prevalent in sequenced samples (14.3\%). Genetic subtyping on the pol gene revealed that the majority of the prisoners were infected with subtype CRF33_01B (52.4\%).

Conclusion: Continuous surveillance of newly infected individuals is required to help strategize the best antiviral treatment for these patients.
\end{abstract}

Key words: HIV-1; CRF33_01B; drug resistance; prisoners.

J Infect Dev Ctries 2014; 8(8):1063-1067. doi:10.3855/jidc.4095

(Received 06 August 2013 - Accepted 23 November 2013)

Copyright (C) 2014 Ariffin et al. This is an open-access article distributed under the Creative Commons Attribution License, which permits unrestricted use, distribution, and reproduction in any medium, provided the original work is properly cited.

\section{Introduction}

Highly active antiretroviral therapy (HAART) was first introduced in Malaysia in 1997 and was chosen to be the preferred treatment strategy from 2001 onward. This resulted in a remarkable reduction of morbidity and mortality rates in HIV-1 patients. HAART, which consists of a combination of two nucleoside reverse transcriptase inhibitors (NRTIs) and a non-nucleoside reverse transcriptase inhibitor (NNRTI) or a protease inhibitor (PI), is accessible through clinics in designated government general and district hospitals $[1]$.

Although HAART is beneficial, increasing its use unfortunately leads to the emergence of drug-resistant HIV strains. Poor adherence to ARV treatments further contributes to the high number of resistant HIV strains reported [2]. Subsequently, this could lead to the transmission of ARV-resistant strains of HIV to susceptible individuals and eventually to a less effective first-line regimen.
HIV-1 subtypes reported to circulate in Malaysia thus far include pure subtype $\mathrm{B}$ and six recombinant forms: CRF01_AE [3], CRF33_01B [3], CRF48_01B [4], CRF52_01B [5], CRF53_01B [6], and CRF54_01B [7]. However, most of the molecular epidemiology studies of HIV-1 in Malaysia were carried out in Kuala Lumpur, and thus available data pertaining to the prevalence of HIV-1 subtypes and HIV primary drug resistance in various risk groups in other states, including Kelantan, is lacking. Kelantan is located on the northeastern coast of Peninsular Malaysia and shares a border on the north with Thailand. Interestingly, Kelantan has one of the highest numbers of newly reported HIV cases in Malaysia. This study aimed to describe for the first time the HIV-1 genetic diversity and the prevalence of transmitted primary drug resistance of HIV-1 based on sequence analyses of the pol gene (PR and RT regions) among treatment-naive prisoners from Kelantan. 


\section{Methodology}

Study population

Twenty-one plasma samples were collected from treatment-naive HIV-1-infected prisoners in Pengkalan Chepa Prison, Kelantan, between June 2009 and January 2012. Clinical data were obtained from prisoners' medical histories. The study protocol was approved by the Research Ethics Committee (Human), Universiti Sains Malaysia (USMKK/PPP/JEPeM198.3[1]). Informed consent was obtained from all patients prior to sample collection.

\section{Genotypic resistance assessment}

The samples were analyzed for HIV-1 resistance mutations in the PR and RT regions. Viral RNA extraction, reverse transcriptase-PCR (RT-PCR), and nested PCR were performed as previously described [8]. Nucleotide sequences of PR and RT regions were edited, aligned, and analyzed for drug resistance using the Stanford HIV Resistance Database (http://hivdb.stanford.edu/).

\section{HIV-1 subtype classification}

Nucleotide sequences were aligned using Clustal $\mathrm{W}$ in the MEGA 4 version 4.0.2 program (Arizona State University, Arizona, USA). The sequences covered all of PR (HXB2 position: 2253-2549) and most of RT (HXB2 position: 2550-3279). In subtype assessment, phylogenetic analyses were performed by the neighbour-joining (NJ) method using Kimura twoparameter distance estimation method with transitiontransversion ratio of 2.0 in 1,000 bootstrap replicates. The recombination form was further analyzed by performing bootscanning analysis with a sliding window of $200 \mathrm{bp}$, incremental steps of 20 bases, and the Kimura two-parameter model using Simplot 3.2 software (Johns Hopkins University, Baltimore, USA).

\section{Statistical analysis}

Fisher's exact test in SPSS version 20 (IBM, New York, USA) was used to evaluate any possible association of HIV-1 subtype with demographic and risk factors.

\section{Sequence data}

Sequences generated have been submitted to GenBank under accession numbers KC762591KC762616.

\section{Results}

\section{Patient baseline characteristics}

Among the 21 prisoners, $14(66.7 \%)$ were males and $7(33.3 \%)$ were females. All prisoners were Malaysians, but of different ethnicities: 19 (90.4\%) were Malays and 1 each was Chinese $(1.4 \%)$ and Indian $(1.4 \%)$. The mean age of the prisoners during sample collection was 39.1 years (standard deviation [SD]: 7.8), and most of them were in the 30-39 $(\mathrm{n}=$ $7,33.3 \%)$ and $40-49(\mathrm{n}=8,38.1 \%)$ age groups. The median of viral load at sampling was $10^{4.76}$ (interquartile range [IR]: $10^{4.86}$ ) RNA copies $/ \mathrm{mL}$. The median CD4 count was 325 (IR: 185) cells $/ \mathrm{mm}^{3}$ (Table 1).

Figure 1. Phylogenetic analysis of 1026 bp HIV-1 pol sequences (HXB2: 2253-3279) among 21 prisoner samples for subtype characterization. HIV-1 reference subtypes and CRFs retrieved from Los Alamos HIV database

(http://www.hiv.lanl.gov/). Reference strains used: subtype B (including Thai B'), CRF01_AE, CRF15_01B, CRF33_01B, CRF34 01B, CRF48 01B, CRF51 01B, CRF52 01B, CRF53 01B, and CRF54 01B. O.C.M.91MVP5180.L20571 was included as an outgroup.

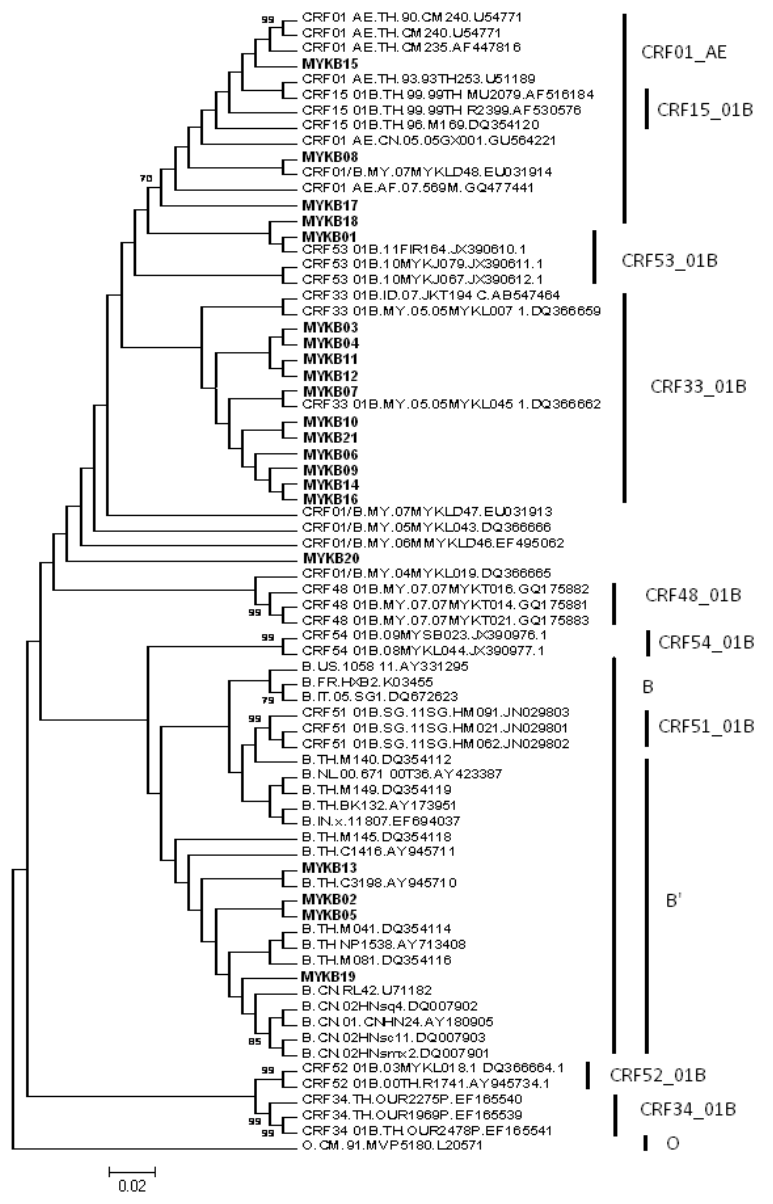


Table 1. Baseline characteristics of treatment-naive HIV-1 infected prisoners

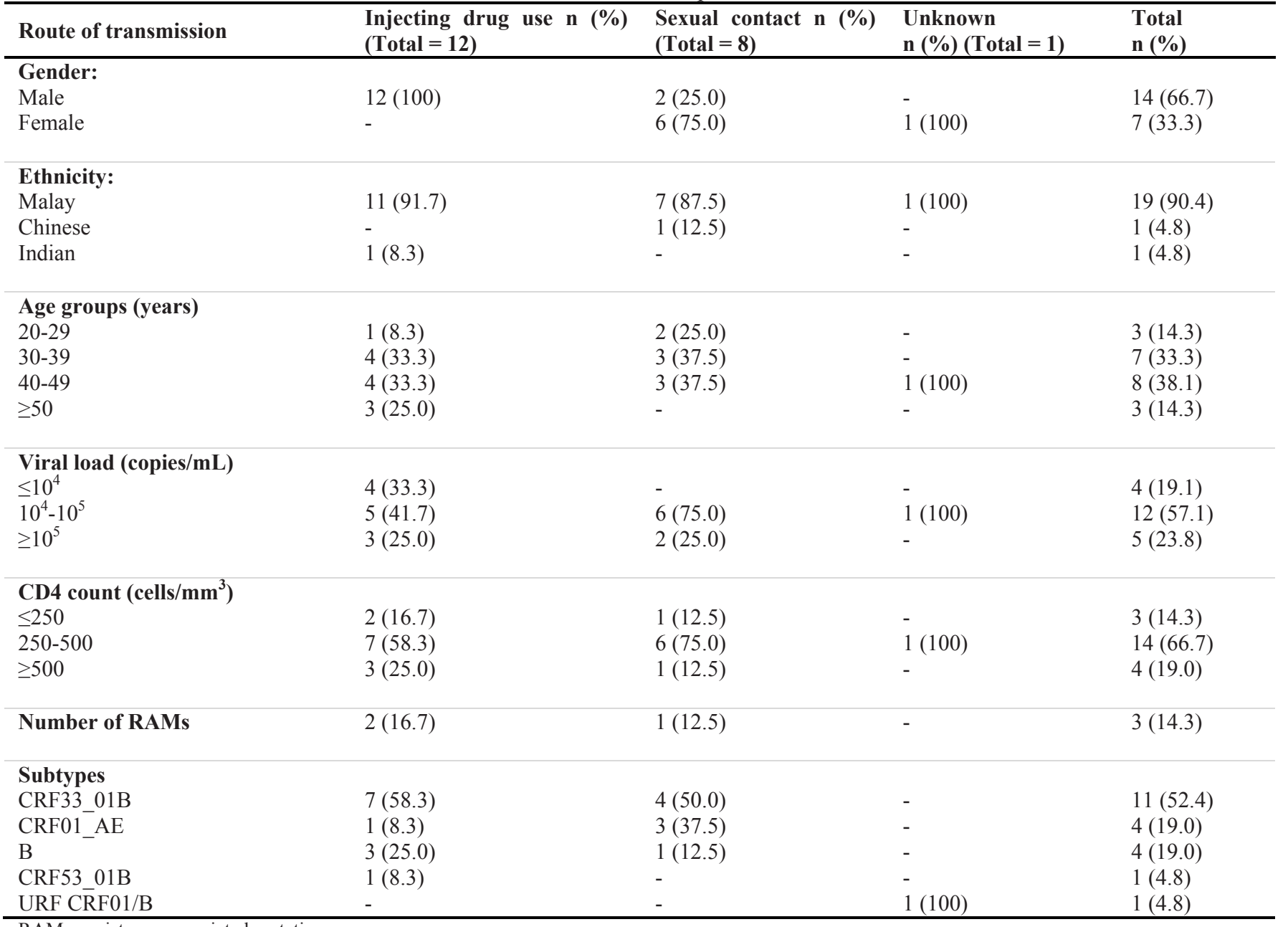

RAMs: resistance-associated mutations

Table 2. HIV-1 genotypes and drug resistance analyses in treatment-naive prisoners in Kelantan

\begin{tabular}{|c|c|c|c|c|c|}
\hline Sample ID & $\begin{array}{ll}\begin{array}{l}\text { Viral } \\
(\text { copies } / \mathrm{mL})\end{array} & \text { load } \\
\end{array}$ & $\begin{array}{ll}\text { CD4 } \\
\left(\text { cells } / \mathrm{mm}^{3}\right)\end{array}$ & Subtype & $\begin{array}{l}\text { NRTI-resistant } \\
\text { mutation }\end{array}$ & $\begin{array}{l}\text { NNRTI-resistant } \\
\text { mutations }\end{array}$ \\
\hline MYKB02 & $10^{4.59}$ & 598 & B' & - & K103N \\
\hline MYKB04 & $10^{4.51}$ & 492 & CRF33_01B & - & - \\
\hline MYKB05 & $10^{3.21}$ & 325 & B' & - & - \\
\hline MYKB06 & $10^{3.74}$ & 389 & CRF33_01B & - & K103N, Y188L \\
\hline MYKB09 & $10^{4.98}$ & 438 & CRF33_01B & - & - \\
\hline MYKB10 & $10^{3.75}$ & 314 & CRF33-01B & - & - \\
\hline MYKB11 & $10^{4.55}$ & 300 & CRF33_01B & - & - \\
\hline MYKB12 & $10^{5.33}$ & 214 & CRF33_01B & - & - \\
\hline MYKB13 & $10^{4.61}$ & 282 & $\mathrm{~B}^{\prime}$ & - & - \\
\hline MYKB14 & $10^{4.81}$ & 595 & CRF33_01B & - & - \\
\hline MYKB19 & $10^{3.78}$ & 633 & $\mathrm{~B}^{\prime}$ & 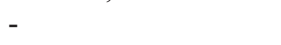 & - \\
\hline MYKB20 & $10^{4.27}$ & 358 & CRF01 AE/B & - & - \\
\hline MYKB21 & $10^{5.59}$ & 222 & CRF33 01B & - & - \\
\hline
\end{tabular}


The major mode of transmission was intravenous drug use (IDU) (57.1\%), followed by sexual contact $(38.1 \%)$. The majority $(85.7 \%)$ of male prisoners acquired HIV infection through IDU ( $p<0.001)$, while $85.7 \%$ of female prisoners acquired it through sexual contact $(\mathrm{p}<0.001)$.

Fisher's exact test analysis revealed no significant association between HIV-1 subtypes and gender, race, and route of transmission ( $\mathrm{p}>0.05)$ (data not shown).

\section{Drug resistance profile}

Only three prisoners $(14.3 \%)$ were resistant to at least one ARV drug; one prisoner was resistant to both NRTIs and NNRTIs, and the other two prisoners were resistant to NNRTIs only. Resistance to PI drugs, however, was not observed. In the RT region, one prisoner had multiple NRTI resistance-associated mutations: M41L, T69N, L74I, M184V, and T215Y (Table 2). For NNRTI resistance-associated mutations, $\mathrm{K} 103 \mathrm{~N}$ was the most prevalent $(\mathrm{n}=3,14.3 \%)$, followed by V179T, Y181C, and Y188L, with a prevalence of $4.8 \%(n=1)$ each. The total number of resistance-associated mutations (RAMs) to NNRTIs and NRTIs was six and five, respectively.

\section{Subtype distribution}

Based on phylogenetic analysis of the pol gene (PR-RT region), CRF33 01B was the most prevalent subtype among the prisoners; $52.4 \%(\mathrm{n}=11)$ harbored this subtype of HIV-1 (Figure 1). This was followed by subtype $\mathrm{B}^{\prime}$ (Thai strain) $(\mathrm{n}=4,19.0 \%)$, CRF01_AE $(\mathrm{n}=4,19.0 \%)$, CRF53_01B $(\mathrm{n}=1$, $4.8 \%$ ), and CRF01_AE/B unique recombinant form (URF) $(\mathrm{n}=1,4.8 \%)$. Interestingly, CRF33_01B also predominated in both prisoners who acquired infection through injecting drugs $(58.3 \%, 7 / 12)$ and those who acquired it through sexual contact $(50.0 \%, 4 / 8)$. Moreover, CRF33_01B also predominated in both genders; $57.1 \%$ of males and $37.5 \%$ of females harboured this subtype.

\section{Discussion}

The present study reveals that RAMs exist in treatment-naive HIV-1 infected persons; similar findings have been reported by others [9-14]. The prevalence of RAMs in the present study $(14.3 \%)$ is in agreement with studies done in Italy $(12.5 \%)$ and the United States (16.0\%) [9-10]. However, in this study, the drug resistance assessment was limited to the pol region since entry and integrase inhibitors are not widely used in HIV patients in Malaysia.
Mutations conferring high resistance to NRTIs were found in an HIV-1 sequence isolated from a female prisoner, MYKB18. This sequence bore thymidine analogue mutations (TAMs) M41L and $\mathrm{T} 215 \mathrm{Y}$, and 69 insertion complex (T69N), causing a high level of resistance to six NRTI drugs except tenofovir [15]. The female prisoner acquired this highly resistant virus from her husband. However, since there were no further data regarding treatment and ARV mutation status of prisoner MYKB18's husband, the actual source of these transmitted drug resistance mutations was unknown.

RAMs for NNRTIs were detected in three prisoners: $\mathrm{K} 103 \mathrm{~N}$ in prisoner MYKB02, K103N and Y188L in prisoner MYKB06, and K103N and Y188C in prisoner MYKB18. However, these prisoners harboured different subtypes; MYKB02 had subtype B', MYKB06 had CRF33 01B, and MYKB18 had CRF01_AE (Table 2). K103N mutation was the most common RAM presented among treatment-naive patients. This finding corroborates several other studies that reported that $\mathrm{K} 103 \mathrm{~N}$ was highly prevalent among NNRTI-associated resistance mutations [1112] or among RAMs in treatment-naive patients [1314]. K103N mutation causes high resistance to nevirapine and efavirenz [16], and thus limits the available choice of the best drug combination in firstline treatment for patients.

Recombinant form CRF33 01B, which was first reported in Kuala Lumpur in 2006 , was found to be the major HIV-1 subtype circulating among prisoners in this study. Previously, it was shown that CRF01_AE was the most prevalent subtype in Malaysia [3]. However, in 2007, a rapid expansion of CRF33_01B in Kuala Lumpur was observed, suggesting a possible replacement of predominant subtypes in near future [17]. Interestingly, in 2012, Mohamad et al. (2012) [8] reported that 44.4\% of pediatric patients harboured subtype CRF33_01B. The following year, CRF33_01B was found to be highly prevalent among drug users (71\%) [18]. The finding of this present study further substantiates the observed shifting trend of the current predominant HIV-1 subtype from CRF01_AE to CRF33_01B in various risk groups in Malaysia. It is interesting to note that CRF53_01B, which was first reported to have emerged in Kuala Lumpur in 2012, was also found in this study (albeit in one prisoner), suggesting possible geographical expansion of this particular subtype. 


\section{Conclusions}

The present study reported for the first time the transmitted drug resistance mutation among treatmentnaive prisoners in Malaysia. Drug resistance to NNRTIs was identified in $3 / 21(14.3 \%)$ isolates subtype B', CRF01_AE, and CRF33_01B. The study also found that CRF33 01B was the most common subtype among treatment-naive prisoners in Kelantan.

\section{Acknowledgements}

This study was supported by the USM Research University grant (1001/PPSP/8120209) and Esciencefund grant (305/PPSP/6113215). Tengku Ahmad Akram Tengku Mohd Ariffin was a recipient of Universiti Sains Malaysia Fellowship.

\section{References}

1. Malaysian Society of Infectious Diseases and Chemotherapy (2001) Consensus on antiretroviral treatment: 2nd national guidelines on antiretroviral therapy, 2nd edition. Kuala Lumpur: Ministry of Health Malaysia. $49 \mathrm{p}$.

2. Valle-Bahena OM, Ramos-Jiménez J, Ortiz-López R, Revol A, Lugo-Trampe A, Barrera-Saldaña HA, Rojas-Martínez A (2006) Frequency of protease and reverse transcriptase drug resistance mutations in naive HIV-infected patients. Arch Med Res 37: 1022-1027.

3. Tee KK, Li XJ, Nohtomi K, Ng KP, Kamarulzaman A, Takebe Y (2006) Identification of a novel circulating recombinant form (CRF33_01B) disseminating widely among various risk populations in Kuala Lumpur, Malaysia. J Acquir Immune Defic Syndr 43: 523-529.

4. Li Y, Tee KK, Liao H, Hase S, Uenishi R, Li XJ, Tsuchiura T, Yang R, Govindasamy S, Yong YK, Tan HY, Pybus OG, Kamarulzaman A, Takebe Y (2010) Identification of a novel second-generation circulating recombinant form (CRF48_01B) in Malaysia: A descendant of the previously identified CRF33_01B. J Acquir Immune Defic Syndr 54: 129-136.

5. Liu Y, Li L, Bao Z, Li H, Zhuang D, Liu S, Wang X, Li T, Jia L, Yang S, Li J (2012) Identification of a novel HIV type 1 circulating recombinant form (CRF52 01B) in Southeast Asia. AIDS Res Hum Retroviruses 28: 1357-1361.

6. Chow WZ, Al-Darraji H, Lee YM, Takebe Y, Kamarulzaman A, Tee KK (2012) Genome sequences of a novel HIV-1 CRF53 01B identified in Malaysia. J Virol 86: 11398-11399.

7. Ng KT, Ong LY, Takebe Y, Kamarulzaman A, Tee KK (2012) Genome sequence of a novel HIV-1 circulating recombinant form 54_01B from Malaysia. J Virol 86: 1140511406 .

8. Mohamad S, Deris ZZ, Yusoff NK, Ariffin TAATM, Shueb RH (2012) Assessing subtypes and drug resistance mutations among HIV-1 infected children who failed antiretroviral therapy in Kelantan, Malaysia. Braz J Infect Dis 16: 284-288.

9. García-Guerrero J, Hoya PSDL, Portilla J, Marco A, SánchezPayá J, Moreno S (2006) Prevalence of HIV-1 drug resistance mutations among Spanish prison inmates. Eur J Clin Microbiol Infect Dis 25: 695-701.

10. Stone DR, Corcoran C, Wurcel A, McGovern B, Quirk J, Brewer A, Sutton L, Richard TD (2002) Antiretroviral drug resistance mutations in antiretroviral-naive prisoners. Clin Infect Dis 35: 883-886.
11. García F, Pérez-Cachafeiro S, Guillot V, Alvarez M, PérezRomero P, Pérez-Elías MJ, Viciana I, Blanco JR, LópezDieguez M, de Mendoza C, Cohort of the Spanish AIDS Research Network (CoRIS) (2011) Transmission of HIV drug resistance and non-B subtype distribution in the Spanish cohort of antiretroviral treatment naïve HIV-infected individuals (CoRIS). Antiviral Res 91: 150-153.

12. Pérez L, Kourí V, Alemán Y, Abrahantes Y, Correa C, Aragonés C, Martínez O, Pérez J, Fonseca C, Campos J, Álvarez D, Schrooten Y, Dekeersmaeker N, Imbrechts S, Beheydt G, Vinken L, Soto Y, Álvarez A, Vandamme A-M, Laethem KV (2013) Antiretroviral drug resistance in HIV-1 therapy-naive patients in Cuba. Infection, Genetics and Evolution 16: 144-150.

13. Gonsalez CR, Alcalde R, Nishiya A, Barreto CC, Silva FES, de Almeida A, Mendonc M, Ferreira F, Fernandes SS, Casseb J, Duarte AJS (2007) Drug resistance among chronic HIV-1infected patients naive for use of anti-retroviral therapy in Sao Paulo city. Virus Res 129: 87-90.

14. Hamers RL, Wallis CL, Kityo C, Siwale M, Mandaliya K, Conradie F, Botes ME, Wellington M, Osibogun A, Sigaloff KCE, Nankya I, Schuurman R, Wit FW, Stevens WS, Vugt MV, de Wit RTF, for Pharm Access African Studies to Evaluate Resistance (PASER) (2011) HIV-1 drug resistance in antiretroviral-naive individuals in sub-Saharan Africa after rollout of antiretroviral therapy: a multicentre observational study. Lancet Infect Dis 11: 750-759.

15. Johnson VA, Calvez V, Günthard HF, Parades R, Pillay D, Shafer RW, Wensing AM, Richman DD (2013) Special contribution: update of the drug resistance mutations in HIV1: March 2013. Top Antivir Med 21: 4-12.

16. National Agency for AIDS Research (ANRS)- AC 11: Resistance Group Version number 22 September 2012. http://www.hivfrenchresistance.org/. Accessed 14 May 2013.

17. Wang B, Lau KA, Ong LY, Shah M, Steain MC, Foley B, Dwyer DE, Chew CB, Kamarulzaman A, Ng KP, Saksena NK (2007) Complex patterns of the HIV-1 epidemic in Kuala Lumpur, Malaysia: evidence for expansion of circulating recombinant form CRF33_01B and detection of multiple other recombinants. Virology 367: 288-297.

18. Chow WZ, Ong LY, Razak SH, Lee YM, Ng KT, Yong YK, Azmel A, Takebe Y, Al-Darraji HAA, Kamarulzaman A, Tee KK (2013) Molecular diversity of HIV-1 among people who inject drugs in Kuala Lumpur, Malaysia: massive expansion of circulating recombinant form (CRF) 33 01B and emergence of multiple unique recombinant clusters. PloS one 8: e62560.

\section{Corresponding author}

Rafidah Hanim Shueb

Department of Medical Microbiology \& Parasitology

School of Medical Sciences, Health Campus

Universiti Sains Malaysia

16150 Kubang Kerian, Kelantan, Malaysia

Phone: +6097676255

Fax: +6097676289

Email: hanimshueb@gmail.com

Conflict of interests: No conflict of interests is declared. 